\title{
THIN FILMS AND HETEROSTRUCTURES OF LAYERED OXIDES: PREPARATION AND PROPERTIES
}

\author{
P. Przyslupski \\ Institute of Physics, Polish Academy of Sciences \\ Al. Lotników 32/46, 02-668 Warszawa, Poland
}

\begin{abstract}
A brief overview of the deposition and properties of $\mathrm{YBa}_{2} \mathrm{Cu}_{3} \mathrm{O}_{7}(\mathrm{YBCO})$ based heterostructures is presented. Emphasis is placed on the assessment of the deposition process and the properties of practical thin films and heterostructures of layered oxides. Besides a reference to the literature we present our recent results concerning deposition, structural and magnetic properties of $\mathrm{YBa}_{2} \mathrm{Cu}_{3} \mathrm{O}_{7} / \mathrm{RE}_{1-x} \mathrm{~A}_{x} \mathrm{MnO}_{3}$ (YBCO/REMO) heterostructures.

PACS numbers: 74.72.Bk, 74.76.-w, 75.70.Cn
\end{abstract}

\section{Introduction}

The discovery of high transition temperature superconductivity (HTSC) in copper oxides renewed interest in employing superconducting thin films in basic research and application. Utilization of this potential requires a well-controlled reliable superconducting thin films technology and heterostructures composed of materials with metallic, semiconducting, insulating, ferroelectric or magnetic properties. Several types of YBCO based heterostructures were prepared such as $\mathrm{YBCO} / \mathrm{PrBCO}$ [1-3], YBCO/NdCeCuO [4], DyBaCuO/SrCaRuO [5], YBCO/ $\mathrm{BaTiO}[6,7]$ and YBCO/REMO [8-10].

More recently, a ferroelectric type of $(\mathrm{Pb}, \mathrm{Ba}, \mathrm{Sr})(\mathrm{Ti}, \mathrm{Zr}) \mathrm{O}_{3}$ [11-13] compounds has been extensively studied because of the potential application in non-volatile memory devices, dielectric capacitors and electro-optics. For such devices a large remanent switchable polarization of ferroelectric material is used. Optical and electric properties and the Curie point can be tailored by partially replacing the $\mathrm{Ba}^{2+}$ ions by $\mathrm{Sr}^{2+}$ ions over a broad range of $x$ from 0 to 1 . In oxide perovskites mobile oxygen and related defects are believed to play an important role in electrical properties.

The discovery of high temperature superconductivity in hole doped copper oxides has renewed interest in $\mathrm{RE}_{1-x} \mathrm{~A}_{x} \mathrm{MnO}_{3}$ (REMO) systems for which a giant magnetoresistance transport (GMR) effect have been reported. An extremely large GMR effect has been measured in LaCaMnO [14, 15] and NdSrMnO [16] thin 
films. In these compositions the GMR effect occurs naturally. The origin of GMR effects is not well established. However, it is well known that metal-semiconductor transitions spontaneously occur at temperatures below such transitions. The substitution of $\mathrm{RE}=\mathrm{La}$ or $\mathrm{Nd}$ ions by di-valent ions $\mathrm{A}=\mathrm{Ca}, \mathrm{Sr}$ or $\mathrm{Ba}$ creates in the transition metal oxides a mixed valence state for $\mathrm{Mn}$, i.e. $\mathrm{Mn}^{3+}$ and $\mathrm{Mn}^{4+}$. It is believed that for certain concentrations a ferromagnetic interaction takes place between these ions through a double exchange interaction [17]. This fact explains the coexistence of ferromagnetism and metallic like conductivity in the perovskite compounds. Electronic properties and structures of such strongly correlated electron systems such hole doped Mn oxides, therefore critically depend on the band filling. That is why, in both above discussed cases the preparation conditions such as, deposition temperature, partial oxygen pressure, post annealing process, etc., will have a strong influence on their electronic properties.

In this paper we present results on the structural and magnetic properties of YBCO/LCMO and YBCO/NSMO heterostructures. Such systems should be useful for studying the influence of decoupling on flux line lattice dynamics between YBCO sublayers by the "spacer" with ferromagnetic properties.

\section{Experiment}

Thin films and heterostructures were prepared by high pressure d.c. sputtering $[3,9,10]$ method from stoichiometric $\mathrm{YBa}_{2} \mathrm{Cu}_{3} \mathrm{O}_{7}, \mathrm{La}_{0.67} \mathrm{Ca}_{0.33} \mathrm{MnO}_{3}$ and $\mathrm{Nd}_{0.68} \mathrm{Sr}_{0.33} \mathrm{MnO}_{3}$ targets. The deposition of the films was carried out on (100) $\mathrm{SrTiO}_{3}$ and (100) $\mathrm{LaAlO}_{3}$ substrates using a platinum strip heater, whose temperature was set at $900^{\circ} \mathrm{C}$ to $930^{\circ} \mathrm{C}$. This corresponds to a temperature of the substrate of about $750^{\circ} \mathrm{C}$. After deposition the films were cooled to about $595^{\circ} \mathrm{C}$ and kept at this temperature for about $20 \mathrm{~min}$. The phase composition and the structure of the deposited heterostructures and thin films were determined using $\mathrm{X}$-ray diffraction ( $\mathrm{Co} K_{\alpha}$ ). Temperature and field dependence of the magnetic moment have been measured using a SQUID magnetometer.

\section{Results}

In Fig. 1 the measurements of the resistance as a function of temperature of $[\mathrm{YBCO}(48 \AA) / \mathrm{LCMO}(60 \AA)]_{14}$ and $[\mathrm{YBCO}(70 \AA) / \mathrm{LCMO}(40 \AA)]_{12}$ multilayers are presented. For thicker YBCO sublayers the resistance above $T_{\mathrm{c}}$-onset shows metallic-like behaviour, whereas for thinner YBCO the resistance above $T_{\mathrm{c}}$-onset is semiconductor-like, i.e. dominated by the LCMO system. In both cases below $T_{\mathrm{c}}$-onset the resistance does not reach zero, similar as it was observed for the trilayer structure [18]. Such a residual resistance below $T_{\mathrm{c}}$-onset suggests some degree of interdiffusion between the YBCO and LCMO sublayers, presumeably of $\mathrm{Ca}$ into YBCO.

In Fig. 2a the relation of the magnetic moment vs. magnetic field is plotted showing a typical hysteresis curve of a ferromagnetically ordered material. Figure 2b presents the zero field cooled (ZFC) and field cooled (FC) magnetic moment of the LCMO film at 800 Oe in a wide temperature range.

In Fig. 3a measurements of the magnetic moment vs. magnetic field for YBCO/LCMO multilayer show diamagnetism for the magnetic field below $100 \mathrm{Oe}$ 


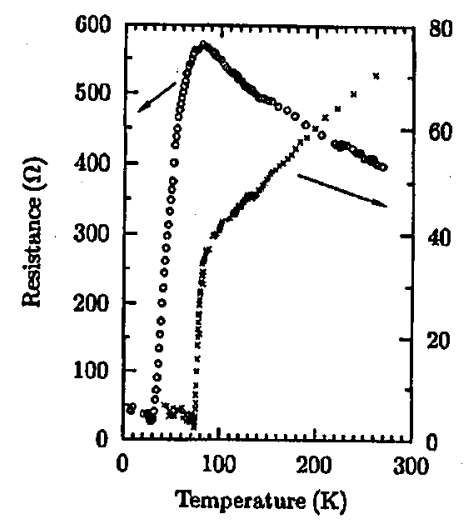

Fig. 1. Resistance transitions for $[\mathrm{YBCO}(18 \AA) / \mathrm{LACMO}(60 \AA)]_{14}(0)$ and $[\mathrm{YBCO}(70 \AA) / \mathrm{LCMO}(60 \AA)]_{12}(\times)$ multilayers.
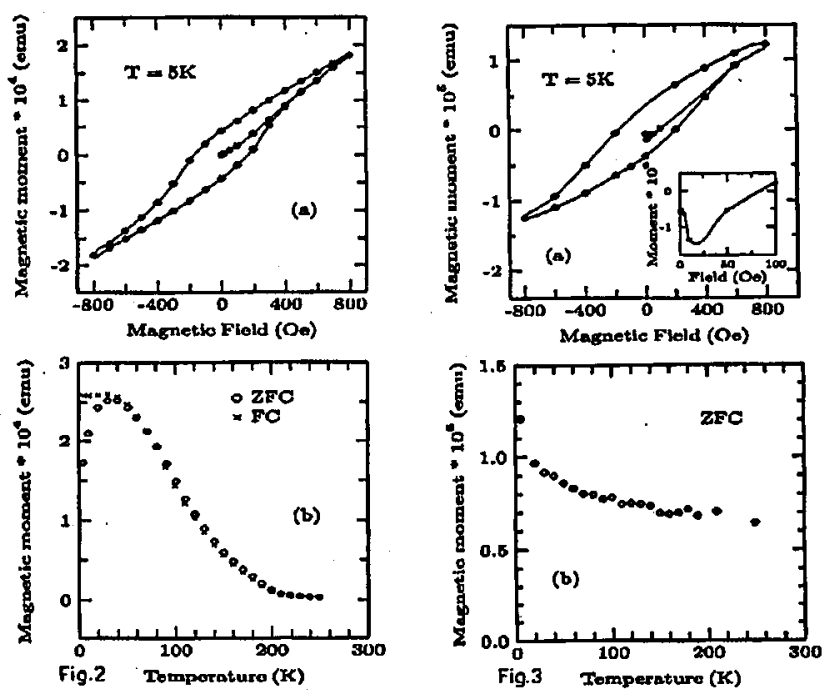

Fig. 2. (a) Magnetic moment of LCMO film as a function of magnetic field ( $\boldsymbol{H} \|$ substrate) and (b) ZFC and FC magnetic moment vs. temperature in a magnetic field of $50 \mathrm{Oe}$.

Fig. 3. (a) Magnetic moment vs. magnetic field ( $H \perp$ substrate) for $[\mathrm{YBCO}(70 \AA) /$ LCMO $(40 \AA)$ ] multilayer, data in enlarged scale are shown in the inset. (b) ZFC magnetic moment of the multilayer vs. magnetic field of $800 \mathrm{Oe}$.

and ferromagnetic order in higher fields. This result indicates the coexistence of superconductivity and ferromagnetic order in the total volume of the multilayer.

In order to avoid interdiffusion we have chosen to study another system, i.e. a YBCO/NSMO. In Fig. 4 an X-ray diagram of a NSMO film, a YBCO/NSMO 

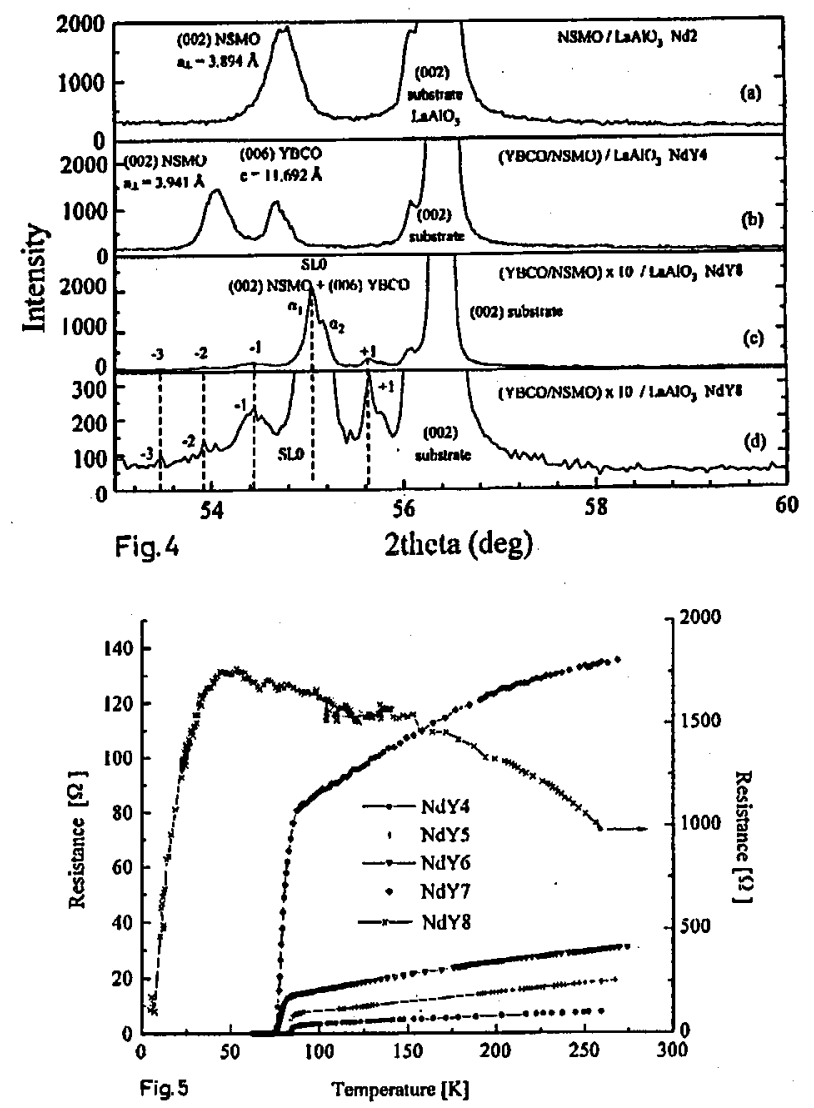

Fig. 4. X-ray diffraction pattern along the (001) axis for (a) NSMO film, (b) the YBCO/NSMO bilayer and (c, d) the YBCO/NSMO superlattice.

Fig. 5. Resistance transitions of the measured YBCO/NSMO heterostructures at critical temperature.

bilayer and YBCO/NSMO superlattice is presented. These results show epitaxial growth of NSMO films. For the YBCO/NSMO superlattice third order satellites are seen indicating a good structural order. We have observed that the lattice parameter of the NSMO films depends on their thickneses. Such a behaviour is due to some differences in the lattice parameters of the $\mathrm{LaAlO}_{3}$ substrate $(a=3.79 \AA)$ and NSMO system. The X-ray analysis of the bulk NSMO compound shows a perovskite-like structure with the lattice parameter $a=3.86 \AA$.

Based on the X-ray diffraction pattern we have obtained the lattice parameter for the sample Nd2 $a_{\perp}=3.894 \AA$ (perpendicular to the substrate). Assuming that the volume of the NSMO unit cell is the same in bulk as in a thin film form we have calculated the lattice parameter parallel to the substrate surface to be $a_{\|}=3.843 \AA$. Similar considerations for the YBCO/NSMO bilayer give $a_{\perp}=3.941 \AA$ and $a_{\|}=3.82 \AA$, respectively. The resistance transitions for the 


\section{TABLE}

Modulation length, thickness and zero resistance of the measured samples.

\begin{tabular}{l|l|c|l}
\hline \hline Sample No. & Modulation (thickness) & $T_{\text {co }}[\mathrm{K}]$ & Remarks \\
\hline Nd1 & NSMO $(1500 \AA)$ & - & film \\
Nd2 & NSMO $(1400 \AA)$ & - & film \\
NdY4 & $\mathrm{YBCO}(1200 \AA) / \mathrm{NSMO}(1000 \AA)$ & 86 & bilayer \\
NdY5 & {$[\mathrm{YBCO}(400 \AA) / \mathrm{NSMO}(320 \AA)]_{2}$} & 78 & multilayer \\
NdY6 & {$[\mathrm{YBCO}(192 \AA) / \mathrm{NSMO}(166 \AA)]_{6}$} & 75 & multilayer \\
NdY7 & {$[\mathrm{YBCO}(69 \AA) / \mathrm{NSMO}(166 \AA)]_{8}$} & 70 & superlattice \\
NdY8 & {$[\mathrm{YBCO}(23 \AA) / \mathrm{NSMO}(166 \AA)]_{10}$} & - & superlattice
\end{tabular}

samples presented in Table are shown in Fig. 5. Only the superlattice NdY8 shows a semiconductor-like behaviour and some residual resistance below $T_{\mathrm{c}}$-onset. Such a behaviour could be due to some imperfection of the interface between the YBCO and NSMO sublayers. In Fig. 6 we present the field dependence of the magnetic moment of a NSMO thin film at two different temperatures. The magnetic moment shows the hysteresis typical for a ferromagnetically ordered material.

Magnetic moment vs. magnetic field data of YBCO/NSMO superlattice are presented in Fig. 7. The shape of the $m(H)$ curve is a result of a superposition of superconducting and ferromagnetic hysteresis curves. This result confirms the coexistence of superconductivity and ferromagnetic order in the total volume of the heterostructure, where superconductivity is associated with the YBCO sublayer and ferromagnetism with the NSMO sublayer.
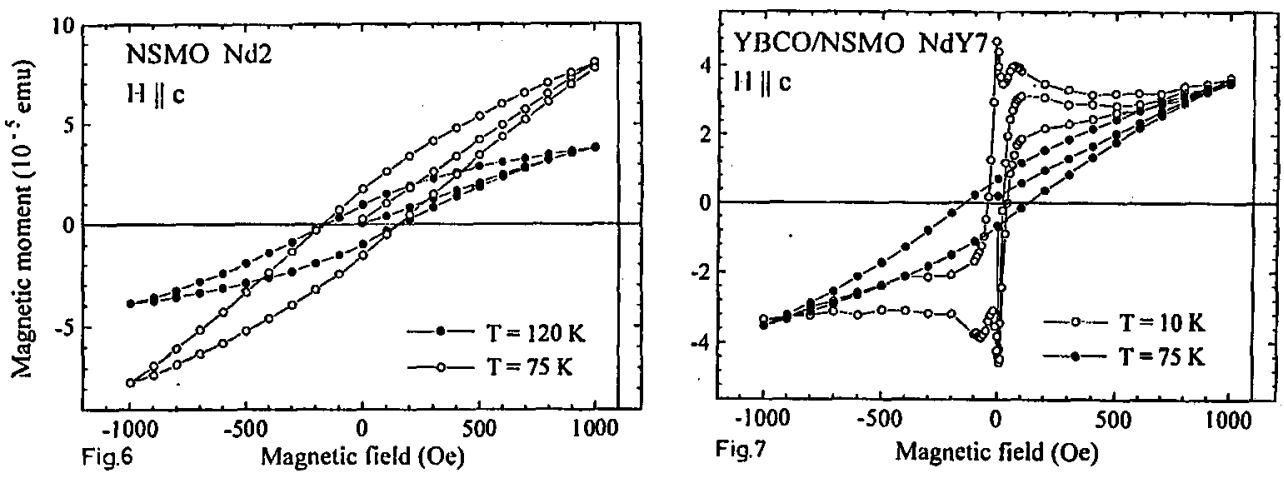

Fig. 6. Magnetic moment of a NSMO thin film vs. magnetic field applied parallel to the $c$-axis.

Fig. 7. Magnetic moment vs. magnetic field of the YBCO/NSMO superlattice applied parallel to the $c$-axis. 
This result indicates the coexistence of both counteracting phenomena on submesoscopic scale (perpendicular to the substrate surface) in the total volume of the samples.

In some way it resembles the results of measurements of $\mathrm{HoMo}_{6} \mathrm{~S}_{8}$ films [19]. Although the mechanism of coexistence in both systems is different the response of the YBCO/NSMO heterostructure to the external magnetic field is the same, i.e. below the superconducting transitions the system shows a ferromagnetic order. In $\mathrm{IIoMo}_{6} \mathrm{~S}_{8}$ compound the superconducting state is associated with $d$-electrons $(\mathrm{Mo})$ and the ferromagnetic order with $f$-electrons (Ho), however below the so-called re-entrant transition a domain-type coexistence is possible. In the case of YBCO/NSMO heterostructures the superconducting state is associated with the YBCO sublayer and the ferromagnetic order with the NSMO sublayer.

In summary we have prepared YBCO/REMO superlattices with a good structural order. Magnetic measurements show a coexistence of superconductivity and the ferromagnetic order in the total volume of heterostructures, where the superconducting state is related to the YBCO sublayers and the ferromagnetic order to the REMO sublayers.

\section{Acknowledgments}

We thank to Drs. E. Dynowska and S. Koleśnik for the experimental help and the Committee for Scientific Research(KBN) for financial support under grant No. 2P30213705.

\section{References}

[1] J.M. Triscone, M.G. Karkut, L. Antognaza, O. Bruner, O. Fischer, Phys. Rev. Lett. 63, 1016 (1989).

[2] Q. Li, X. Xi, X.D. Wu, A. Inam, S. Vadlamanati, V.L. McLean, T. Ventkatesan, R. Ramesh, D. Hwang, J.A. Martinez, L. Nazar, Phys. Rev. Lett. 64, 3086 (1990).

[3] G. Jakob, P. Przysłupski, C. Stolzel, C. Tome Rosa, A. Walkenhorst, M. Schmitt, H. Adrian, Appl. Phys. Lett. 59, 1626 (1991).

[4] A. Gupta, R. Gross, E. Olsson, A. Segmuller, G. Koren, C.C. Tsuei, Phys. Rev. Lett. 64, 3191 (1990).

[5] L. Mieville, E. Koller, J.M. Triscone, M. Decroux, O. Fischer, Phys. Rev. B, to be published.

[6] R. Ramesh, W.K. Chan, B. Wilkens, II. Gilchrist, T. Sands, J.M. Tarascon, V.G. Keramides, D.K. Fork, J. Lee, A. Safari, Appl. Phys. Lett. 61, 1537 (1992).

[7] J. Lee, L. Johnson, A. Safari, R. Ramesh, T. Sands, H. Gilchrist, V.G. Keramidas, Appl. Phys. Lett. 63, 27 (1993).

[8] G. Jakob, V.V. Moshalkow, Y. Bryunseraede, Appl. Phys. Lett. 66, 2564 (1995).

[9] P. Przysłupski, A. Wiśniewski, R. Szymczak, J. Igalson, Czech. J. Phys. 46, 1355 (1996).

[10] P. Przysłupski, S. Koleśnik, E. Dynowska, T. Skośkiewicz, M. Sawicki, presented at ASC'96 Conf., to be published in IEEE Appl. Supercond.

[11] J.F. Scott, C.A. Paz de Arauja, Sciences 246, 1400 (1989).

[12] E. Dey, H. Zulee, Ferroelecttrics 108, 37 (1990). 
[13] J. Lee, R. Ramesh, V.G. Keramidas, W.L. Warren, G.E. Pike, J.T. Evans, Appl. Phys. Lett. 66, 1337 (1995).

[14] S. Jin, T.H. Tiefel, M. McCormack, R.A. Fastnacht, R. Ramesh, L.H. Chen, Science 264, 413 (1993).

[15] R. von Helmolt, J. Wecker, B. Holtzapfel, L. Schultz, K. Samwer, Phys. Rev. Lett. 71, 2331 (1993).

[16] G.C. Xiang, Q. Li, H.L. Ju, S.N. Mao, L. Senapatii, X.X. Xi, R.L. Greene, T. Ventkatesan, Appl. Phys. Lett. 66, 1427 (1995).

[17] G.H. Jonker, J.H. van Santen, Physica 16, 737 (1950).

[18] K. Chachara, T. Okuno, M. Kasai, Y. Kanake, Y. Kozono, Appl. Phys. Lett. 62, 780 (1993).

[19] P. Przysłupski, U. Poppe, K. Fischer, Ch. Buchal, Z. Phys. 59, 407 (1985). 